\title{
A Model of Multi-Domain Clustering for Local Network in Modern Vehicles
}

\author{
E. Yu. Pavlenko, ${ }^{*}$ K. S. Vasilyeva, ${ }^{\dagger}$ and P. D. Zegzhda ${ }^{\ddagger}$ \\ Peter the Great St. Petersburg Polytechnic University, \\ 29 Politechnicheskaya Str, 195251 Saint-Petersburg, RUSSIA
}

(Received 14 July, 2020)

\begin{abstract}
The paper reviews prospects of using domain architecture as a basis for implementing the car network security mechanism, based on the ECU authentication. It contains an analysis of the existing methods of domain formation and proposes a method for a comprehensive assessment of the security classes of car's components, taking into account, in addition to the functional purpose of the ECU, a list of its interfaces, interaction with other ECUs, the difference between integrity levels of slave devices. Also it proffers a network clustering approach based on combining elements of one security class into a domain.
\end{abstract}

AMS Subject Classification: 00A06, 05C05, 05C20

Keywords: vehicle cybersecurity, connected cars, controller area network, domain architecture, cluster-tree, integrity level, fingerprinting ECU

DOI: https://doi.org/10.33581/1561-4085-2020-23-3-280-290

\section{Introduction}

Ensuring the cybersecurity of a modern motor vehicle (MV), which is a complex cyberphysical system that actively cooperates with the environment and provides mechanisms for autopilot and remote control, requires an integrated approach that includes the development of a flexible model of permits [1-3]. The model performs control of network communication of architectural components of the MV and is able to adapt to the expansion of multiple points of communication of the MV with third-party infrastructure elements. However, the implementation of such a model is impossible without the mechanism of identification and authentication of electronic control units (ECUs) which interact within the local network of the vehicle. Application of individual identification methods is complicated by the need to equip an absolute set of MV units with the appropriate mechanism, which is not always possible due to the following factors [4-7]:

- limited computing power of some ECU;

\footnotetext{
*E-mail: pavlenko@ibks.spbstu.ru

${ }^{\dagger}$ E-mail: vasilieva.kv@edu.spbstu.ru

${ }^{\ddagger}$ E-mail: zeg@ibks.spbstu.ru
}

- limited payload size provided by network protocols, which are in use in cars network;

- stringent real-time performance requirements.

\section{Related Works}

One of the methods to overcome these problems is the transition to the domain architecture. Within this architecture it can be organized group identification, which allows to reduce the load on the network and relocate the MV network security functionality in the gateway nodes without requiring modification of the builtin software of existing ECU.

Methods of clustering the internal MV network are based on the formation of several large domains. These domains are distributed ECU in accordance with their functional purpose. Primarily, five domains are allocated [8-12]:

- the infotainment system domain includes a navigation service, components for playing various types of multimedia, a module of communication with a mobile device and so on; 
- the driver and passenger comfort domain includes such modules as adaptive cruise control, climate control, seat adjustment system, interior air cleaning;

- the chassis control domain combines modules that directly influence traffic control (e.g. steering and braking) and emergency safety components (e.g. airbag systems);

- the transmission control domain includes management systems of: engine, suspension, automatic transmission, stabilization system, etc;

- the body control domain is responsible for the various components of access to the MV (power operated door locks, anti-theft systems, keyless access), as well as auxiliary services that provide control of the external and internal lighting of the MV, wipers, direction-indicator lamps and alarm lamps.

Such differentiation is relevant for relatively simple tasks that do not require a flexible configuration. For example, to make a decision on the complete isolation of the domain (eg, information and entertainment system) or to form a list of ECU, for which you should prohibit the update "by air". However, for a modern MV the division into these domains is not constructive due to the following reasons:

1. Domain isolation on the principle of wireless communication interfaces is impossible, because such a solution leads to the inoperability of a significant number of functional components of remote interaction with the MV: from remote engine start and pre-conditioning to unmanned parktronic and any new generation of V2X-communications.

2. Separation only on the basis of functional category does not take into account the level of exposure of each particular ECU to external influences (e.g. the presence of wireless communication interfaces) and does not provide an estimate of the potential damage to which the ECU will be compromised. This results in combining components with different integrity levels that have different security ensuring requirements into a single domain. For example, both the alarm, central locking and immobilizer control module and the luggage compartment lid control component are classified as the body control domain.

3. Domain architecture provides for the location on the border of each cluster of the gateway implementing some protective mechanism (IPS/IDS/DPI, addition to the frame with identification data, application of lightweight cryptographic protocols and algorithms [13], etc.). In this case, if components of different integrity levels are located in the domain, the only effective solution is to assign to the entire domain the integrity level of the most critical component. For example, for the body control domain, the total level of required protection will be determined by the security alarm control ECU, but security measures will also be applied to the control lid luggage compartment ECU. Such redundancy leads to unjustified increase of network load and traffic passing through the gateway node, which means that it can negatively affect the components' performance under conditions of limited computing power, which is unacceptable from the point of view of correct functioning of the MV real-time system.

4. The architecture of modern MV provides for the presence of several central control units, which control a lot of different directions ECU, which makes it difficult to unambiguously assign such a central unit to a certain functional domain.

Thus, ensuring the security of new generation vehicles by forming protected domains requires the development of a method of flexible automated clustering of the vehicle's 
Table 1: Risk ranking.

\begin{tabular}{|c|c|}
\hline \hline Interface type & Weight \\
& $W_{i}=2 * W_{i-1}, W_{0}=100$ \\
\hline driver distraction, $R_{0}$ & 100 \\
\hline sensitive data leak, $R_{1}$ & 200 \\
\hline car damage, $R_{2}$ & 400 \\
\hline creating/exacerbating an emergency, $R_{3}$ & 800 \\
\hline car theft, $R_{4}$ & 1600 \\
\hline loss of control, $R_{5}$ & 3200 \\
\hline
\end{tabular}

Table 2. Calculation of functional risk indices.

\begin{tabular}{|c|c|c|c|c|c|c|c|}
\hline Risk & $\begin{array}{l}\text { loss of } \\
\text { control }\end{array}$ & car theft & $\begin{array}{c}\text { creating / } \\
\text { exacerbating an } \\
\text { emergency }\end{array}$ & $\begin{array}{c}\text { car } \\
\text { damage }\end{array}$ & $\begin{array}{l}\text { sensitive } \\
\text { data leak }\end{array}$ & $\begin{array}{c}\text { Driver } \\
\text { distraction }\end{array}$ & \\
\hline Category & $\mathrm{W}_{5}=3200$ & $\mathrm{~W}_{4}=1600$ & $\mathrm{~W}_{3}=800$ & $\mathrm{~W}_{2}=400$ & $\mathrm{~W}_{1}=200$ & $\mathrm{~W}_{0}=100$ & \\
\hline Active control & 1 & 1 & 1 & 1 & 0 & 0 & 6000 \\
\hline $\begin{array}{l}\text { Chassis and } \\
\text { transmission }\end{array}$ & 1 & 1 & 1 & 1 & 0 & 0 & 6000 \\
\hline $\begin{array}{l}\text { Active driver } \\
\text { assistance }\end{array}$ & 1 & 0 & 1 & 1 & 0 & 0 & 4400 \\
\hline $\begin{array}{l}\text { Anti-theft } \\
\text { system }\end{array}$ & 0 & 1 & 0 & 0 & 0 & 1 & 1700 \\
\hline $\begin{array}{l}\text { Passive } \\
\text { security }\end{array}$ & 0 & 0 & 1 & 1 & 0 & 1 & 1300 \\
\hline $\begin{array}{l}\text { Ensuring of } \\
\text { external } \\
\text { visibility }\end{array}$ & 0 & 0 & 1 & 1 & 0 & 0 & 1200 \\
\hline $\begin{array}{l}\text { Light and } \\
\text { sound alarms }\end{array}$ & 0 & 0 & 1 & 1 & 0 & 0 & 1200 \\
\hline $\begin{array}{c}\text { Control of } \\
\text { body and seat } \\
\text { components }\end{array}$ & 0 & 0 & 0 & 1 & 0 & 1 & 500 \\
\hline Navigation & 0 & 0 & 0 & 0 & 1 & 1 & 300 \\
\hline $\begin{array}{l}\text { Infotainment } \\
\text { system }\end{array}$ & 0 & 0 & 0 & 0 & 1 & 1 & 300 \\
\hline $\begin{array}{l}\text { Climate } \\
\text { control }\end{array}$ & 0 & 0 & 0 & 0 & 0 & 1 & 100 \\
\hline $\begin{array}{c}\text { Car interior } \\
\text { lighting }\end{array}$ & 0 & 0 & 0 & 0 & 0 & 1 & 100 \\
\hline
\end{tabular}


local network, which is based on a comprehensive assessment of the characteristics of the ECU, including the features of its interaction with other components and its location in the existing topology.

\section{Proposed method description and risks evaluation}

The choice of levels dividing devices into classes was made according to the key types of computer attacks on modern vehicles. These types of attacks are correlated with the five domains of the ECU, resulting in the division, taking into account not only the functions of the devices, but also possible damage due to ECU compromise.

In this research paper it is proposed to set the Safety Integrity Level (SIL) of ECU as the main characteristic value, on the basis of which the decision about the scope of measures to ensure the security of the respective ECU is made, and to establish the dependence of this value on the following characteristics of the ECU:

- functional risk index, R;

- risk scattering index, $\mathrm{S}$;

- external exposure index, I;

- internetworking engagement index, $\mathrm{N}$;

- number of backup devices, D.

In the first step, the whole set of ECU is divided into $\mathrm{N}$ functional groups, combining control units of similar purpose and specifying previously defined categories (such as comfort domain, chassis control domain, etc.). Then, for each group is defined a set of theoretically possible risks that occur when an ECU of this group fails or when an intruder gets control over it. The following list of risks is considered:

- driver distraction, $R_{0}$;

- sensitive data leak, $R_{1}$;

- car damage, $R_{2}$;
- creating / exacerbating an emergency, $R_{3}$;

- car theft, $R_{4}$;

- loss of control, $R_{5}$.

The authors chose the multiplier equal to 2 based on the results of their experiments. If necessary, this multiplier can accept other values set by the researcher. Each next level is more important in terms of information security, so for each next level doubling the weight of risk is used. Selecting $W_{0}$ is not fundamental and is done in such a way that the priority of the most important characteristics of the MV is maintained. Table 1 shows the weighting values compared to the given risks at $W_{0}=100$. The general functional risk index $R$ for a given ECU is calculated using the formula $R=\sum_{i=0}^{6} R_{i} * W_{i}$, where $R_{i}$ is a boolean value indicating the presence of the corresponding risk. The initial value of risks was chosen experimentally. The proposed categories of control units and their corresponding functional risk indexes are shown in table 2.

Let us enter the designation "leaf" for those control units that do not have child nodes according to the actual topology of the MV local network. In the next step, each "leaf" control unit is assigned to one of the functional groups and receives the corresponding value of the functional risk index.

After comparison of functional risk $\mathrm{R}$ index to each "leaf" ECU, iterative calculation of similar index for "parent" nodes by the formula is performed:

$$
R_{j}=\max \left(R_{\text {child }_{0}}, R_{\text {child }_{1}}, \ldots, R_{\text {child }_{M}}\right),
$$

where $M$ is the number of $j$-dependent ECU devices. Also for each ECU a risk scattering index is fixed. Risk scattering index is a value that characterizes the degree of heterogeneity of connected "child" nodes and allows to determine the optimal location of the domain boundary:

$$
S_{j}=\left(R_{j}-\min \left(R_{\text {child }_{0}}, \ldots, R_{\text {child }_{M}}\right)\right) M,
$$

where $R_{j}$ is the functional risk index of the $j$-th ECU; $M$ is the number of devices dependent on the $j$-th ECU. 
Table 3: Ranking of third-party connectivity interfaces

\begin{tabular}{|c|c|}
\hline \hline Interface type & Weight \\
& $W_{i}=2 * W_{i-1}, W_{0}=500$ \\
\hline wired, $I_{0}$ & 500 \\
\hline short-range wireless, $I_{1}$ & 1000 \\
\hline long-range wireless, $I_{2}$ & 2000 \\
\hline
\end{tabular}

The next characteristic of an ECU exposure index - is determined depending on the presence of the following standard ECU interfaces with the external environment:

- wired (USB, OBD-II), $I_{0}$;

- short-range wireless (Wi-Fi, Bluetooth), $I_{1}$;

- long-range wireless (GSM, LTE), $I_{2}$.

The exposure index is calculated in the same way as the functional risk index: interfaces are ranked in descending order of opportunities that an attacker must have to use the corresponding interface to gain control over the local MV network. For example, to use USB and OBDII interfaces, an attacker needs to have physical access to the MV, to use Wi-Fi or Bluetooth - to stay at a distance of up to 100 meters, while an attack via an ECU connected to the Internet, theoretically has no territorial restrictions. Table 3 shows the weights compared to the above interface categories at $W_{0}=$ 500. The initial values of the weights were chosen according to interfaces susceptibility to external attack. For example, an ECU attack has no territorial restrictions and is dangerous, so interface $I_{2}$ is assigned the highest weight. The total exposure index $I$ for a given ECU is calculated by the formula $I=\sum_{i=0}^{3} I_{i} W_{i}$, where $I_{i}$ is a Boolean value indicating the presence of the corresponding interface.

The internetworking engagement index is then compared to each ECU, which is calculated on the basis of the bus types to which the control unit in question is connected and the role that is assigned to the unit in case of a "Master-Slave" connection. In general, four bus types are considered:

- Local Interconnect Network (LIN), $N_{0}$;
- Media Oriented Systems Transport (MOST), $N_{1}$;

- Controller area network (CAN), $N_{2}$;

- Flex-Ray, $N_{3}$.

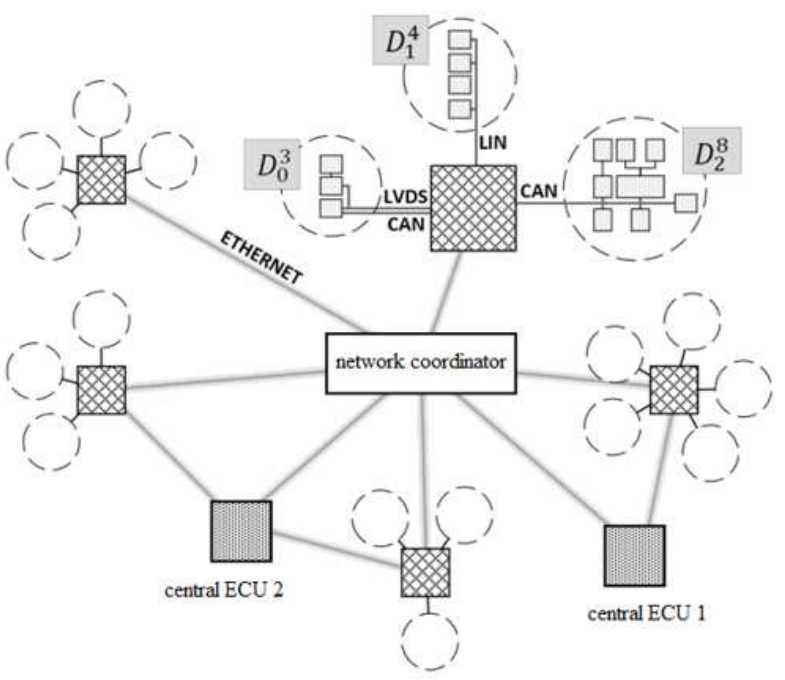

FIG. 1: The general layout of the cluster tree.

\section{Bus types to which the control unit is connected}

The LIN bus is a single-wire serial bus that connects control units within one limited module and provides data rates from 1 to $20 \mathrm{kbps}$. A single master and up to 16 slaves are always connected to this type of bus. The master ECU polls the slaves and sends commands to them; the slaves only respond to requests received, but never initiate them [14]. The role of slave nodes can be 
Table 4: Ranking of MV bus types.

\begin{tabular}{|c|c|}
\hline Interface type & Weight \\
& $W_{i}=2 * W_{i-1}, W_{0}=50$ \\
\hline Local Interconnect Network (LIN), $N_{0}$ & 50 \\
\hline Media Oriented Systems Transport (MOST), $N_{1}$ & 100 \\
\hline Controller area network (CAN), $N_{2}$ & 200 \\
\hline Flex-Ray, $N_{3}$ & 300 \\
\hline
\end{tabular}

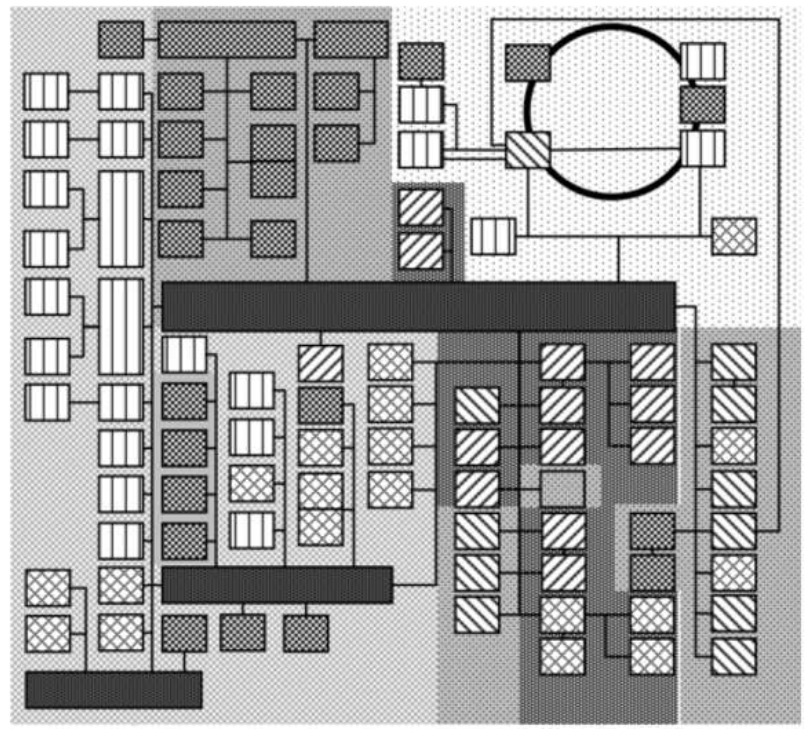

FIG. 2: An example of MV network topology.

played by control units (e.g. fan ECU) as well as sensors and actuators (e.g. gradient sensor or antitheft alarm siren). The LIN bus is often used to connect external controls because its compromise prevents the intruder from gaining control of the local MV network due to the inability to initiate packet sending to the master or other slaves.

The MOST bus is designed for fast data transfer between infotainment system nodes at a speed of up to $150 \mathrm{Mbit} / \mathrm{s}$ (connection of up to 64 devices is supported). The most modern standard MOST-150 enables the organization of an Ethernet channel between devices for packet data transfer. The bus under consideration is distinguished by the presence of an addressing mechanism and a circular structure, in which data frames are moved in series from one device to another in a circle until they return to the nodeaddressant [15]. The MOST bus supports three data channels:
- synchronous channel for audio and video data transmission;

- asynchronous channel for transmitting data packets (TCP/IP) containing, for example, navigation system configuration or control unit software updates;

- service channel for transmitting control commands.

Data transmission is synchronized by the master ECU, whose job is to create fixed frequency frames and assign identifiers to the connected devices. It is important to note that the MOST bus elements are not isolated from other subnetworks: For example, the central ECU of an information system also has the ability to communicate via CAN bus, LVDS line, Ethernet channel and USB interfaces.

CAN-bus is the most universal and widely used for communication between ECU for various purposes. This bus allows transmitting data at a speed of up to $500 \mathrm{Kbit} / \mathrm{s}$ and is bidirectional: any of the connected control units can both transmit and receive messages [16].

The FlexRay bus serves for communication of modules of active security and automatic control of MV (for example, in systems of regulation of dynamics of movement, adaptive cruise control and parktronic autopilot) and provides speed of data transfer to $10 \mathrm{Mbit} / \mathrm{s}$. This bus different determinism, fault tolerance and possibility of functioning in a mode of real time [17]. This bus is characterized by an "active star"topology. In [18] is noted: all ECUs in the FlexRay section are connected to a central control unit, which is called the active node. The role of the active node is often assigned to the bus diagnostic interface to which elements of the 
Table 5. ECUs security classes.

\begin{tabular}{|c|l|c|}
\hline $\begin{array}{c}\text { Security } \\
\text { class }\end{array}$ & \multicolumn{1}{|c|}{ ECU example } & $\begin{array}{c}\text { Hatching on } \\
\text { the topology }\end{array}$ \\
\hline 1 & $\begin{array}{l}\text { Multifunction steering wheel control unit, } \\
\text { automatic transmission control unit, chassis control } \\
\text { unit }\end{array}$ & $\begin{array}{c}\text { Admllmas } \\
\text { control unit }\end{array}$ \\
\hline 2 & $\begin{array}{l}\text { Airbag control unit, alarm siren, anti-theft alarm } \\
\text { sensor, night vision system control unit }\end{array}$ & $\begin{array}{c}\text { Seat and steering column adjustment control unit, } \\
\text { multimedia system control panel, trailer recognition } \\
\text { control unit }\end{array}$ \\
\hline 5 & Air ionizer, interior lighting modules \\
\hline
\end{tabular}

Table 6. ECUs security classes for standard domains without taking into account the integrity level.

\begin{tabular}{|c|c|}
\hline Domain & Background hatching on the topology \\
\hline Infotainment system domain \\
\hline Domain of comfort ensuring & \\
\hline $\begin{array}{c}\text { Chassis control and transmission } \\
\text { control domain }\end{array}$ & \\
\hline Body control domain & \\
\hline
\end{tabular}

chassis domain are connected: control units ABS, motor, steering column, automat transmission, airbags, adaptive cruise control, etc.

None of the buses described generally supports node authentication and payload verification methods, so the ranking of the buses required to calculate the internetworking engagement index is based on the principle of evaluating the capabilities acquired by an attacker when accessing the bus: the widest impact area, including critical components, corresponds to the CAN bus and the FlexRay bus, which provides a smaller footprint but allows communication with components to actively assist the driver; followed by the MOST bus, which does not affect the critical components but participates in the software upgrade process; the lightest weight is the LIN bus, which is mainly used for connecting "leaf" ECUs and allows communication activity to be initiated only if the master device is compromised. Table 4 shows the weights compared to the above interface categories at $W_{0}=50$. Taking into account the given weights, the internetworking engagement index of the $\mathrm{j}$-th ECU is calculated as follows:

$$
N_{j}=\sum_{i=0}^{M} W_{i}-\sum_{i=0}^{K} W_{i}
$$

where $M$ is the total number of buses to which the ECU in question is connected; $K$ is the number of buses to which the ECU is a slave. As part of this research, it is proposed to introduce the following final formula for the integrity level, including the reviewed characteristics of the ECU:

$$
S I L=\frac{R}{D+1}-S+I+N
$$

where $R$ is the functional risk index; $D$ is the number of backup devices; $S$ is the risk scattering index; $I$ is the exposure index; $N$ is the internetworking engagement index.

\section{Algorithm of the proposed method}

Once the integrity level is calculated for each ECU, domains are formed according to the following algorithm: 
1. The obtained values of integrity levels are sorted in descending order and the boundaries of security classes are defined: for example, class 1 includes an ECU, which has $S I L_{i}>4000$, class 2 - ECU, which has $2000 \leq S I L_{i}<4000$, and so on.

2. ECUs are ordered by the integrity level within a single bus (the interconnections between ECUs remain unchanged).

3. ECUs are ordered by the integrity level within several equivalent LIN buses connected to a common master (as long as this does not contradict the common ECU data communication logic).

4. Subsets of ECU are allocated within each bus. Elements of the same security class are combined in these subsets.

5. If more than one subset of ECU is connected to the same bus (i.e. control units connected to the bus are of different security classes), each subset is allocated to a separate additional bus.

6. Each bus that connecting control units of one security class forms a security domain, which is designated $D_{i d}^{M}$, where $i d$ is the domain identifier; $M$ is the number of ECU in the domain.

7. The number of domains is minimized by the following rule: $D_{i}^{M}$ can be combined with domain $D_{j}^{N}$ connected to the same bus as $D_{i}^{M}$, if $M \leq \frac{1}{20}$ of the total number of ECU and security class $D_{j}^{N}$ is 1 higher than security class $D_{i}^{M}$. Final domain keeps security class of $\mathrm{j}$-th domain.

8. The resulting intermediate domain topology is transformed into a cluster tree, whose components are set as follows:

- endpoint - domain with a specific security class;

- cluster - domains set of the same security class connected to one central element - gateway node;
- network coordinator - diagnostic interface to which all clusters are connected.

In addition, the topology may also include several control nodes - central control units directly (bypassing the network coordinator) connected to clusters that include subordinate domains. Gateway nodes, control nodes and the coordinator node communicate over high-speed Ethernet links, exchanging frames that within encapsulate CAN, LIN, FlexRay and so on. Each frame leaving the domain is tagged: the gateway node adds a domain ID and signature, thus achieving group authentication, on the basis of which the security policy is further implemented. The gateway node has at least one physical interface for connecting each domain and (in addition to authentication/integrity/signature control functions, etc.) acts as a translator, making inter-cluster communication transparent to the end nodes. The gateway node meets the following requirements:

- has high computing power and hardware capability of parallel processing of data coming to different interfaces;

- supports all network interfaces used by the domain ECU to communicate outside their domain;

- operates on a real-time operating system with proven reliability (e.g., QNX).

The general layout of the cluster tree is shown in Figure 1.

9. Gateway node interfaces configure the security mode depending on the security class of the cluster (e.g. for low security domains, incoming traffic verification is disabled as the risk of sending malicious commands to elements of these domains is an acceptable risk). 


\section{Experimental testing of the proposed method}

The above method of local network clustering was tested on the flagship MVs of premium segment, including the MV 2019, which has a modern set of remote control and active assistance to the driver. The topology of this MV includes 97 elements, and all the many values obtained should be divided into 6 security classes:

- 1 class: $\forall i: S I L_{i} \geq 6000$;

- 2 class: $\forall i: 4000 \leq S I L_{i}<6000$;

- 3 class: $\forall i: 1000 \leq S I L_{i}<2000$;

- 4 class: $\forall i: 500 \leq S I L_{i}<1000$;

- 5 class: $\forall i: 100 \leq S I L_{i}<500$;

- 6 class: $\forall i: S I L_{i}<0$;

The last category - 6 class - contains 3 central ECUs, for which the risk scattering index significantly prevails over other characteristics. This indicates that the degree of fragmentation of security classes of the nodes controlled by these ECU is so high that it is not reasonable to attribute the data of central ECU to this or that domain: it is more rational to provide a differentiated level of security of control units subordinate to them. The following ECU belong to this category in this example:

- the on-board network control unit (e.g. controls both the passenger compartment lighting system and the components that are part of the immobiliser system);

- the comfort system control unit (e.g. responsible for seat adjustment and antitheft system simultaneously);

- diagnostic data bus interface (is the central node of the topology to which most buses are directly connected).
Listed ECU are marked on the topology marked according to security classes (Fig. 2) in solid dark grey color. Examples of security class 1-5 ECUs and their barcodes are shown in Table 5. Figure 2 also shows the distribution of the ECU for standard domains without taking into account the integrity level (Table 6).

This visualization allows to compare the result of standard domain allocation and clustering based on the ECU integrity levels and emphasizes that large functional domains contain ECUs that belong to different security classes and require different security mechanisms.

The process of domain architecture formation according to the algorithm proposed in this study is shown in Figure 3: the first scheme corresponds to the stage of ordering the ECU by the level of integrity within a single bus, the second - within several equivalent LIN-bus connected to a common master device; the third scheme shows the stage of dividing the buses into subbuses, devices which have a common security class; the fourth scheme shows the stage of allocating security domains.

The numbering of blocks in Figures 3 and 4 reflects the order in which the devices are included in the allocated security domains.

The resulting cluster tree shown in Figure 4 includes five clusters (by the number of security classes highlighted) and, accordingly, five gateway ECUs (highlighted by a dotted hatching and bold outline) - so the increase in the number of control units is approximately $5 \%$ of the total, with one gateway accounting for no more than $24 \%$ of the network elements, which suggests the theoretical possibility of evenly distributed traffic between clusters. 


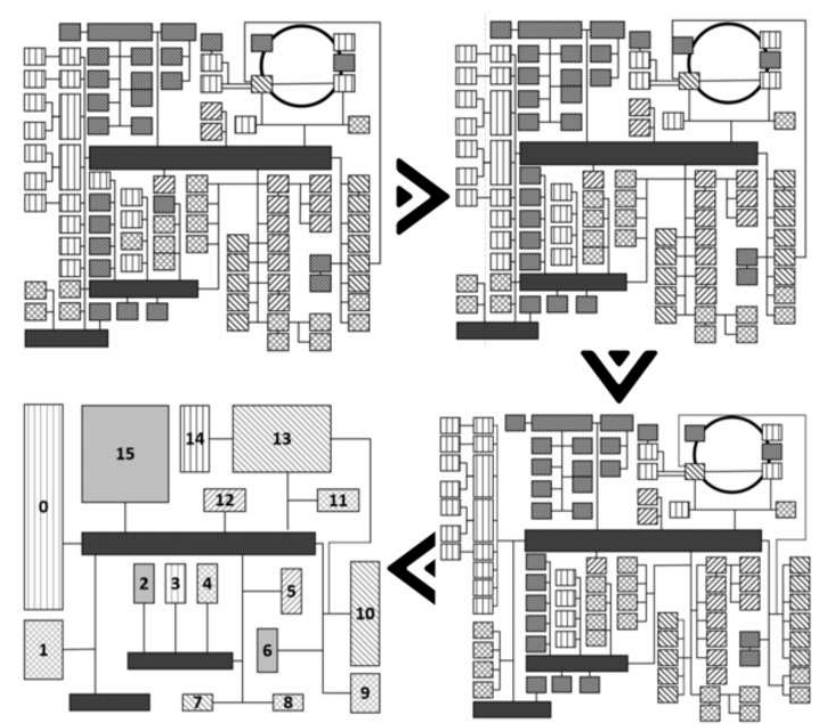

FIG. 3. Example of domain architecture formation process.

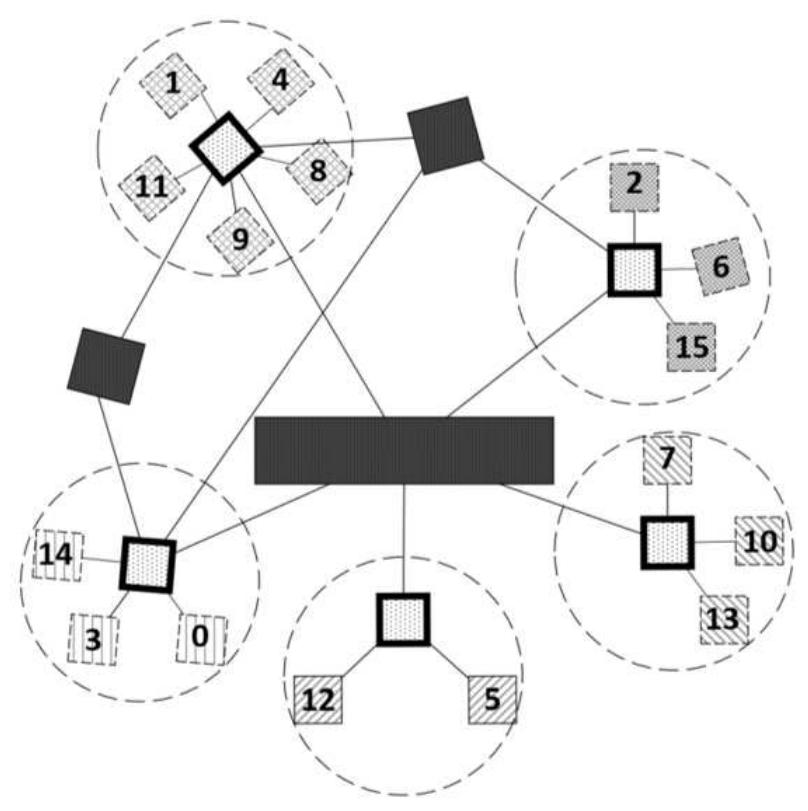

FIG. 4: Example of the final cluster tree.

\section{Conclusion}

Method of organization of the local network of a MV proposed in this paper provides a basis for implementation of a hierarchical model of permits, preventing attacks on the MV of new generation due to its following features:

1. Allows for domain level authentication, which allows for the use of both primitive access restriction tools such as white-list and more complex mechanisms based on credentials/disclosure security policy.

2. Distinguishes itself by its modularity and scalability.

3. Does not impose restrictions on computing resources of existing ECU.

4. Minimizes redundancy of security measures taken.

It also provides flexibility of configuration and possibility to set a single universal set of security rules for the whole cluster, as well as to configure domain security individually (which is especially relevant for domains of high criticality, which include modules of wireless communication with third-party devices).

\section{Acknowledgements}

The study was carried out as a part of a scholarship of the President of the Russian Federation to young scientists and graduate students SP-1689.2019.5.

\section{References}

[1] D. Lavrova, M. Poltavtseva, and A. Shtyrkina, in 2018 IEEE Industrial Cyber-Physical Systems (ICPS) (IEEE, 2018), pp. 818-823.
[2] Y. S. Vasilev, D. P. Zegzhda, and M. A. Poltavtseva, Automatic Control and Computer Sciences 52, 1090 (2018). 
[3] D. P. Zegzhda, Y. S. Vasilev, and M. A. Poltavtseva, Automatic Control and Computer Sciences 52, 1000 (2018).

[4] K.-T. Cho and K. G. Shin, in 25th $\{$ USENIX\} Security Symposium (\{USENIX\} Security 16) (2016), pp. 911-927.

[5] M. Kneib and C. Huth, INFORMATIK 2017 (2017).

[6] W. Choi, H. J. Jo, S. Woo, J. Y. Chun, J. Park, and D. H. Lee, IEEE Transactions on Vehicular Technology 67, 4757 (2018).

[7] H. M. Song, H. R. Kim, and H. K. Kim, in 2016 international conference on information networking (ICOIN) (IEEE, 2016), pp. 63-68.

[8] R. Currie, Retrieved March 17, 2017 (2016).

[9] D. K. Nilsson, P. H. Phung, and U. E. Larson (2008).

[10] McKinsey and Company, (2018, November). Automotive software and electronics $2030 . \quad$ https://www.gsaglobal.org/wpcontent/uploads/2018/12/8.-GSA-MeetingTaipei-Discussion-AGENDA-14-Nov-2018.pdf
(2018).

[11] M. Scalas and G. Giacinto, in 2019 2nd International Conference on new Trends in Computing Sciences (ICTCS) (IEEE, 2019), pp. $1-6$.

[12] https://industrytoday.com/wpcontent/uploads/2018/12/Upstream-SecurityGlobal-Automotive-Cybersecurity-Report2019.pdf (2019).

[13] E. B. Aleksandrova, Automatic Control and Computer Sciences 51, 947 (2017).

[14] E. Hackett, http://www.ti.com/ lit/an/slla383/slla383.pdf (2018).

[15] A. Sumorek and M. Buczaj, Teka Komisji Motoryzacji i Energetyki Rolnictwa 14 (2014).

[16] S. Tuloki and I. Nagy, Múszaki Tudományos Közlemények 9, 243 (2018).

[17] F. Consortium et al., Flexray communication systems protocol specification, version 3.0. 1 [ol] (2010).

[18] K. Anjan, R. Arpitha, P. Keya, and K. Arti, Int. J. Engin. Research \& Technol. 3, 2278 (2014). 